Serrano Riaño Julieth Yadira, ${ }^{1}$ Núñez Camargo Danny Williams, ${ }^{2}$ Najar Céspedes Andrea Paola. ${ }^{3}$

${ }^{1}$ Bacterióloga- MSc-Ciencias Microbiología. Docente Investigadora Fundación Universitaria del Área Andina. jserrano@areandina.edu.co ngeniero agrónomo- MScGestión ambiental. Docente Investigador Fundación Universitaria del Área Andina. dnunez2@areandina.edu.co BBacterióloga- MSc-Ciencias Microbiología. Docente Universidad Militar Nueva Granada. andreanajar@ gmail.com

Trabajo recibido: 21 de junio 2018. Aprobado: 20 de setiembre 2018.

\section{CONDICIONES HIGIÉNICO SANITARIAS DEL \\ MUNICIPIO DE CAPARRAPÍ COLOMBIA Y APARICIÓN DE ENFERMEDAD DIARREICA AGUDA EN LA POBLACIÓN DE 0 A 15 AÑOS.}

CLEANNESS AND SANITARY CONDITIONS OF THE TOWN OF CAPARRAPI- COLOMBIA AND THE APPEARANCE OF ACUTE DIARRHEAL DISEASE IN THE POPULATION BETWEEN $O$ AND 15 YEARS OLD

CONDIÇÕES SANITÁRIAS NO MUNICÍPIO DE CAPARRAPI-COLÔMBIA E O APARECIMENTO DE DOENÇAS DIARREICAS AGUDAS NA POPULAÇÃO DE O A 15 ANOS.

\section{Resumen:}

Este estudio identificó las condiciones higiénico-sanitarias del Municipio y la aparición de enfermedades gastrointestinales (EDA) en la población de 0 a 15 años. Se realizó un estudio cuantitativo descriptivo de corte transversal, se aplicó una encuesta a 90 viviendas y se usó el estadístico Chi2 y el riesgo o Odds ratio para determinar relación de las variables con la aparición de diarrea en la población de 0 a 15 años.En las viviendas encuestadas habitan 349 personas, 116 (33\%) corresponden a 0-15 años; se reportaron 66 casos de Enfermedad Diarreica Aguda (EDA) y de estos el $66.7 \%$ correspondieron a la población de 0-15 años.; las variables presencia del servicio de aseo municipal en el hogar, ubicación del tanque de almacenamiento, disposición de la cocina en el hogar, lavaplatos separado de otros espacios y disposición de excretas fueron las que se relacionaron estadísticamente con la aparición de diarrea en esta población.

Palabras Clave: Salud ambiental, condiciones sanitarias, diarrea. 


\section{Abstract}

This study identified the cleanness and sanitary conditions of the town and the appearance of gastrointestinal diseases in the population between 0 and 15 years old. A cross sectional quantitative descriptive study was done; a survey was applied in 90 homes and Chi Square Statistics and Odds ratio were used to determine the relationship of variables with the appearance of diarrhea in the population between 0 and 15 years old. Three hundred forty nine people live in the homes where the survey was applied; $116(33 \%)$ are between $0-15$ years old; 66 cases of Acute Diarrheal Disease (ADD) were reported and $66.7 \%$ of them correspond to the population aged $0-15$. The presence of municipal cleaning service, location of the storage tank, location of the kitchen within the home, sink separated from other areas and disposition of excreta were the variables statistically related with the appearance or diarrhea in this population.

Key words: Environmental health, sanitary conditions, diarrhea.

Resumo

Este estudo identificou as condições higiênico-sanitárias do município e o aparecimento de doenças gastrointestinais (EDA) na população de 0 a 15 anos. Um estudo transversal descritivo quantitativo foi realizado pesquisando com enquetes em 90 casas, empregando-se o estatístico X2 e a taxa de risco de Odds para determinar a relação variáveis com a ocorrência de diarreia entre a população de $0-15$ años. As moradias pesquisadas são habitadas por 349 pessoas, 116 (33\%) correspondem a 0-15 anos; 66 casos de Doença Diarreica Aguda (D

DA) foram relatados e destes $66,7 \%$ corresponderam à população de $0-15$ anos; as variáveis presença do serviço municipal de limpeza em casa, a localização da caixa d'água, o esquema de cozinha em casa, máquina de lavar louça isolada de outros espaços e eliminação de detritos foram aqueles estatisticamente associados com a ocorrência de diarreia nesta população.

Palavras chave: Saúde ambiental, condições sanitárias, diarréia.

\section{Introducción}

Según un informe presentado por la Organización Mundial de la Salud en el año 2012 perdieron la vida 12.6 millones de personas por vivir o trabajar en ambientes poco saludables, esto representa cerca de la cuarta parte de la totalidad de muertes; así mismo se afirma que factores de riesgo como contaminación del aire, el agua y el suelo, la exposición a los productos químicos, el cambio climático y la radiación ultravioleta, contribuyen a más de 100 enfermedades o traumatismos (1).

Las enfermedades con mayor carga que se pueden atribuir a los factores ambientales son la diarrea, infecciones respiratorias inferiores, otras lesiones y la malaria ocasionando el $26 \%$ de las defunciones en niños menores de 5 años (2). Esta morbilidad es causada por factores ambientales en los países en desarrollo, esto se debe al tipo de exposición a los riesgos ambientales y el difícil acceso a la atención primaria, así mismo se informó que las enfermedades diarreicas debidas a contaminación ambiental aportan cerca de 846000 muertes anuales(1).

Para el año 2002 la OMS estimó que el 88\% de todos los casos globales de diarrea fueron efecto del agua insegura y a la contaminación de los alimentos, así como también de la falta de infraestructura sanitaria, falta de manejo adecuado de excretas, malas prácticas de higiene. Su transmisión puede ocurrir en el domicilio, la escuela, áreas de recreación, 
suelos contaminados con heces (2). En Colombia, la enfermedad diarreica aguda ocupa el segundo lugar de morbi-mortalidad en la población menor de cinco años, especialmente en los municipios con mayor porcentaje de necesidades básicas insatisfechas(3). A su vez en un estudio realizado en el 2002 donde se registró el perfil epidemiológico de una población desplazada en Cartagena se observó que las condiciones higiénico sanitarias en esa población están directamente relacionadas con el aumento en la aparición de diarrea en un 30\% en menores de 5 años comparado con lo reportado sobre las consultas del país que estuvo entre el 9 y 10\% (4). Por otro lado, un estudio realizado en el 2007 acerca de las condiciones sanitarias de los servicios de alimentación en instituciones infantiles en Bucaramanga resalta la importancia de tener un manejo adecuado de los residuos sólidos para evitar la alteración y descomposición de los alimentos que afecten la salud de los infantes (5).

El municipio de Caparrapi tiene una población cercana a los 17 mil habitantes, en este tipo de municipios, el tema de la cobertura de agua potable y alcantarillado es deficiente para las comunidades, (6). La mayor morbilidad presentada en el municipio está relacionada en la primera infancia por problemas gastrointestinales y respiratorios; las infecciones respiratorias representan el $34.6 \%$, el parasitismo intestinal $12.3 \%$, diarrea y gastroenteritis $10.6 \%$ y la bronquitis aguda $3.5 \%$ son las principales causas de consulta, constituyéndose como los principales problemas de salud pública del municipio (7). En la edad de 6 a 15 años, las infecciones respiratorias representan el principal problema (62\%), seguido por parasitosis intestinal (14.2\%) y en menor proporción, las infecciones de vías urinarias (5.3 \%). (7).

\section{Métodos}

Se usó un tipo de estudio cuantitativo observacional descriptivo, de corte transversal. La población de estudio fueron los habitantes del área urbana del municipio de Caparrapí-Cundinamarca. El muestreo: Se utilizó un tipo de muestreo probabilístico aleatorio simple (M.A.S) para la aplicación de la encuesta estandarizada, el número de viviendas se determinó mediante el supuesto del número de hogares en la cabecera del municipio para el 2010 (817) según reportes del DANE de 2005. Se consideró un error de muestreo (v) del 5\%, el tamaño de muestra requerido para este tipo de muestreo fue de 89 viviendas de las 817 mencionadas. El procedimiento: Se encuestaron a 90 hogares de la cabecera municipio utilizando la encuesta estandarizada de vivienda saludable adoptada por la Organización Panamericana de Salud y adaptada por el Ministerio de Protección Social para Colombia (8). Esta encuesta de 124 preguntas evalúa 6 criterios: Control de calidad de la encuesta, datos generales, encuesta psicosocial, vivienda, morbilidad sentida e instrumentos de observación (seguridad y entorno de la vivienda y condiciones de vivienda). Sin embargo, para esta investigación se eligieron las preguntas pertinentes que responden a los objetivos de investigación, las variables se indican a continuación:

Saneamiento básico: Se evaluaron cuatro factores: el primero fue el agua para consumo humano evaluado en las preguntas: ¿De dónde toman principalmente el agua para consumir en la vivienda?, ¿Ustedes obtienen el agua de esta forma con qué regularidad? ¿En que almacenan el agua para consumo humano?, en caso de usar tanque o recipiente ¿Dónde está ubicado?, ¿Cada cuánto se lava?, ¿Tienen tapa?, ¿Qué hacen con el agua antes de consumirla? El segundo factor se refería al manejo de excretas, basuras y residuos sólidos en la vivienda, evaluado en preguntas como: ¿El servicio sanitario es exclusivo o compartido con otras familias?, ¿Cuál es la disposición final de las basuras en este hogar?, ¿Cómo disponen las excretas?, ¿Cómo recogen las basuras?, para definir si el manejo era adecuado o inadecuado se tuvo en cuenta el manejo de basuras por medio de recipientes con tapa, disposición de excretas en inodoro conectado a alcantarillado, sanitario de uso exclusivo, 
recolección de basuras por parte del servicio de aseo municipal, entre otras.

En el tercer factor correspondiente a la manipulación de alimentos, se tuvo en cuenta: sitio de preparación, material de la mesa de preparación, lavado y almacenamiento de los alimentos. Finalmente, el cuarto factor se refería específicamente a factores protectores y condiciones de la vivienda como: aseo en la vivienda, material de las paredes, pisos y techo y disposiciones de algunos lugares dentro de la vivienda como la cocina, el inodoro, el lavamanos etc.

Síntomas y signos de enfermedad enfermedad diarreica aguda: Se utilizó el aparte de morbilidad sentida de la encuesta de vivienda saludable; este instrumento define la aparición de enfermedad diarreica aguda (EDA) mediante la pregunta ¿El menor ha sufrido en el último mes de diarrea o soltura de estómago?, para el presente estudio el periodo en la aparición de EDA se modificó a la aparición de síntomas en los últimos 6 meses descartando posibles picos por periodos de sequía y lluvia en el municipio.

Posible relación del manejo de agua y basuras y alimentos con la enfermedad gastrointestinal en el municipio: Para realizar este aparte se escogieron las viviendas en donde había casos de EDA en personas de 0 a 15 años y se describieron las variables concernientes a los cuatro factores nombrados anteriormente, comparándolas con las viviendas donde residían personas de 0 a 15 años y donde no hubo aparición de diarrea en los últimos 6 meses. Para determinar la relación entre variables medidas con la aparición de diarrea, se aplicó el estadístico Chi 2usando el Software SPSS, se tomó como referencia el $\mathrm{P}$ valor, si este corresponde a un valor menor a 0.05 se acepta la hipótesis alternativa de que existe relación entre las variables y se rechaza hipótesis nula de que no existe relación entre las variables. Para determinar el riesgo o Odds ratio o el tamaño del efecto, se usó el software SPSS calculándolo a un intervalo de confianza al 95\%, este se considera significativo cuando el valor del odds ratio está dentro del intervalo y este supera la unidad Análisis de la información.

\section{Resultados}

Se encontraron un total de 349 habitantes con un promedio de 3.9 personas por vivienda. En cuanto a la edad el mayor grupo de población se encuentra en el grupo de 0-15 años con un 33,2\% (116/90), Se encontró que de las 349 personas el $55 \%$ corresponde al género femenino mientras que el $45 \%$ del género masculino. De las 116 personas correspondientes al rango de edad de $0-15$ se observó que 38 personas tienen entre 0 y 5 años, 43 entre 6-10 años y 35 entre 11-15 años. En cuanto al saneamiento básico se analizaron cuatro factores: agua para el consumo básico, se observó como factor de riesgo que el servicio del acueducto es irregular e intermitente con un $47 \%$ así mismo el almacenamiento de agua en tanques sin tapa con un $17 \%$ y una frecuencia de lavado de los mismos muy heterogénea siendo lo ideal tres veces al año, sin embargo, se observa que cerca de un $23 \%$ no cumplen con lo recomendado).

En cuanto al manejo de excretas y basuras se observa que la mayoría lo hacen a través del servicio de aseo municipal, llama la atención que cerca del $11 \%$ de las viviendas usan pozos sépticos para disposición de las heces, lo que puede aumentar el riesgo de infección. (9-11). En cuanto a los factores de riesgo y factores protectores de la familia, el uso de elementos de protección como toldillos, mallas y fumigación es importante ya que el municipio posee gran población de plagas y vectores; Por otra parte el tener la ropa almacenada en sitios adecuados y separada por cada miembro de la familia con un $87 \%$ reduce el riesgo de infección cuando uno de los miembros la tiene alguna enfermedad infecto contagiosa, previniendo la distribución de agentes infecciosos a través de fómites. (12). (Ver Tabla 1). 
Tabla 1. Saneamiento Básico de las viviendas encuestadas en el municipio de Caparrapi.

\begin{tabular}{|c|c|c|c|}
\hline AGUA PARA EL CONSUMO HUMANO & $\underline{\text { SI \% }}$ & $\frac{\text { MANEJO DE EXCRETAS Y MANEJO DE }}{\underline{\text { RESIDUOS SÓLIDOS Y BASURAS }}}$ & $\underline{\text { SI\% }}$ \\
\hline $\begin{array}{l}\text { El agua para consumo proviene del acueducto } \\
\text { público }\end{array}$ & 89 & $\begin{array}{l}\text { El servicio sanitario exclusivo para las personas de } \\
\text { la familia }\end{array}$ & 98 \\
\hline El agua se consume sin tratamiento previo & 19 & $\begin{array}{l}\text { La disposición final de las basuras es por el } \\
\text { servicio de aseo municipal }\end{array}$ & 92 \\
\hline El agua que se toma es embotellada & 21 & $\begin{array}{l}\text { Las excretas se disponen en el inodoro conectado } \\
\text { al alcantarillado }\end{array}$ & 81 \\
\hline $\begin{array}{l}\text { El servicio de acueducto es irregular e } \\
\text { intermitente }\end{array}$ & 47 & Las excretas se disponen en un pozo séptico & 11 \\
\hline $\begin{array}{l}\text { Usan tanques de almacenamiento de agua con } \\
\text { tapa }\end{array}$ & 67 & El lavamanos se encuentra cerca del sanitario & 78 \\
\hline $\begin{array}{l}\text { Usan tanques de almacenamiento de agua sin } \\
\text { tapa }\end{array}$ & 17 & La basura se dispone en bolsas plásticas & 56 \\
\hline $\begin{array}{l}\text { Frecuencia de lavado de tanques de } \\
\text { almacenamiento de agua }\end{array}$ & & La basura se dispone en recipientes con tapa & 21 \\
\hline 2 veces al mes & 17 & La basura se dispone en recipientes sin tapa & 19 \\
\hline $1 \mathrm{vez}$ al mes & 23 & MANIPULACIÓN DE ALIMENTOS & $\underline{\mathrm{SI} \%}$ \\
\hline 1 vez cada $2-5$ meses & 21 & Se cocina en un cuarto aparte destinado para tal fin & 97 \\
\hline Cada 6 meses o mas & 14 & $\begin{array}{l}\text { Lavan las frutas y verduras crudas antes del } \\
\text { consumo }\end{array}$ & 100 \\
\hline Nunca se lava el tanque & 9 & $\begin{array}{l}\text { Material de la mesa en donde se elaboran } \\
\text { alimentos }\end{array}$ & \\
\hline $\begin{array}{l}\text { FACTORES DE RIESGO/ } \\
\text { PROTECTORES DE LA FAMILIA }\end{array}$ & SI\% & Cemento & 31 \\
\hline $\begin{array}{l}\text { Usan elementos para protección contra } \\
\text { plagas y vectores }\end{array}$ & 83 & Madera & 21 \\
\hline $\begin{array}{l}\text { Disponen de la ropa en guardarropas, closet y } \\
\text { roperos }\end{array}$ & 87 & Baldosa & 25 \\
\hline $\begin{array}{l}\text { Separan la ropa de cada miembro de la } \\
\text { familia }\end{array}$ & 87 & Almacenan los alimentos en el refrigerador & 88 \\
\hline $\begin{array}{l}\text { Asean al vivienda con detergentes y } \\
\text { desinfectantes }\end{array}$ & 77 & $\begin{array}{l}\text { Almacenan los alimentos en recipientes cerrados, } \\
\text { abiertos o al aire libre }\end{array}$ & 12 \\
\hline
\end{tabular}

\section{Síntomas y signos de enfermedad gastrointestinal -Morbilidad Sentida}

Se encontraron 66 casos de EDA, con 22 casos en la edad de más de 15 años seguidos con 19 casos en la edad de 0 a 5 años. Se observa una diferencia marcada por la edad constituyendo una afectación mayor (dos veces más) en las edades de $0-15$ años constituido por un $66.7 \%$ frente a un $33.3 \%$ del rango de más de 15 años esto sugiere tal como afirma la literatura (13) que este periodo de edad es el más sensible a presentar enfermedades gastrointestinales, a su vez se observa claramente que la frecuencia de EDA disminuye con la edad. (Tabla 2). 
Tabla $\mathbf{N}^{0}$ 2: Distribución por edad y frecuencia de casos de EDA reportados.

\begin{tabular}{|cccr}
\hline $\begin{array}{c}\text { Edad } \\
\text { (años) }\end{array}$ & $\begin{array}{c}\text { Casos reportados de } \\
\text { EDA (n=66) }\end{array}$ & $\begin{array}{c}\text { Frecuencia (\%) de casos de EDA por } \\
\text { edad respecto a la totalidad de casos } \\
\text { de EDA }(\mathbf{n}=\mathbf{6 6})\end{array}$ & $\begin{array}{c}\text { Frecuencia (\%) de } \\
\text { casos de EDA por edad }\end{array}$ \\
\hline $0-5$ & 19 & $28,8 \%$ & $(19 / 38) 50 \%$ \\
\hline $6-10$ & 17 & $25,8 \%$ & $(17 / 43) 39.5 \%$ \\
\hline $11-15$ & 8 & $12,1 \%$ & $(8 / 35) 23 \%$ \\
\hline Más de 15 & 22 & $33,3 \%$ & $(22 / 233) 6,3 \%$ \\
\hline
\end{tabular}

\section{Relación del manejo de agua y basuras y alimentos con la enfermedad gastrointestinal}

Para el análisis de los datos se tomaron como variables las reportadas en la Tabla. 3 y 4 con esta información de 65 viviendas en donde habitaban personas de 0 a 15 años, se construyeron tablas de contingencia para determinar las frecuencias cruzando las variables de presencia o no de diarrea con las características sanitarias, hábitos de los hogares, para determinar cuál de estos factores tiene relación. Para determinar la relación se aplicó el estadístico Chi 2 usando el Software SPSS, se tomó como referencia el P valor, si este corresponde a un valor menor a 0.05 se acepta la hipótesis alternativa de que existe relación entre las variables y se rechaza hipótesis nula de que no existe relación entre las variables. De las 65 viviendas el $50.8 \%(\mathrm{n}=33)$ presentaron episodios de diarrea en los últimos 6 meses y el 49.2\% $(n=32)$ de las mismas no lo presentaron.

Tabla No 3: Relación de variables concernientes al manejo de agua en el municipio de Caparrapí en viviendas con personas residentes de 0 a 15 años.

\begin{tabular}{|c|c|c|c|c|c|c|}
\hline VARIABLES & Con & Sin & Chi 2 & \multicolumn{3}{|c|}{ Riesgo } \\
\hline Acueducto & $\mathbf{n}$ & $\mathbf{n}$ & $\mathrm{P}<0,05$ & OR & \multicolumn{2}{|c|}{ IC $95 \%$} \\
\hline $\mathrm{Si}$ & 30 & 31 & 0,318 & 0,323 & 0,032 & 3,276 \\
\hline No & 3 & 1 & & & & \\
\hline Uso del sanitario en el hogar & & & 0,746 & & & \\
\hline Uso colectivo & 1 & 1 & & 1,032 & 0,062 & 17,24 \\
\hline Uso exclusivo de la familia & 32 & 31 & & & & \\
\hline Acceso a Alcantarillado & & & 0,102 & & & \\
\hline $\mathrm{Si}$ & 25 & 29 & & 0,323 & 0,077 & 1,352 \\
\hline No & 8 & 3 & & & & \\
\hline Servicio de Aseo & & & $\underline{0,042}$ & N/A & N/A & $\mathrm{N} / \mathrm{A}$ \\
\hline $\mathrm{Si}$ & 29 & 32 & & & & \\
\hline No & 4 & 0 & & & & \\
\hline $\begin{array}{r}\text { Sitio de toma de agua de } \\
\text { consumo }\end{array}$ & & & 0,318 & 3,1 & 0,305 & 31,487 \\
\hline Acueducto & 30 & 31 & & & & \\
\hline Aguas superficiales & 3 & 1 & & & & \\
\hline $\begin{array}{r}\text { Frecuencia de toma de agua } \\
\text { de consumo }\end{array}$ & & & 0,358 & 0,737 & 0,277 & 1,96 \\
\hline Regular & 14 & 16 & & & & \\
\hline Irregular & 19 & 16 & & & & \\
\hline $\begin{array}{r}\text { Almacenamiento de agua de } \\
\text { consumo humano }\end{array}$ & & & 0,418 & 0,825 & 0,283 & 2,406 \\
\hline Tanque con tapa & 24 & 22 & & & & \\
\hline Tanque sin tapa & 9 & 10 & & & & \\
\hline $\begin{array}{l}\text { Frecuencia de lavado del } \\
\text { tanque de almacenamiento }\end{array}$ & & & 0,468 & & & \\
\hline Al menos una vez al año & 24 & 22 & & 0,825 & 0,283 & 2,406 \\
\hline Mas de un año & 9 & 10 & & & & \\
\hline $\begin{array}{r}\text { Ubicación del tanque en el } \\
\text { hogar }\end{array}$ & & & $* 0,014$ & N/A & $\mathrm{N} / \mathrm{A}$ & N/A \\
\hline Adecuado & 10 & 1 & & & & \\
\hline No almacena & 3 & 4 & & & & \\
\hline Inadecuado & 20 & 27 & & & & \\
\hline Agua antes de tomarla & & & 0,525 & 1,167 & 0,345 & 3,945 \\
\hline Con tratamiento & 26 & 26 & & & & \\
\hline Sin Tratamiento & 7 & 6 & & & & \\
\hline
\end{tabular}


Tabla No 4: Relación de variables manejo de excretas, basuras, alimentos y condiciones de viviendacon la aparición de EDA en el municipio de Caparrapí en viviendas con personas residentes de 0 a 15 años.

\begin{tabular}{|c|c|c|c|c|c|c|}
\hline \multirow{3}{*}{\begin{tabular}{|} 
VARIABLES \\
$\begin{array}{r}\text { Disposición final de } \\
\text { las Basuras }\end{array}$
\end{tabular}} & \multirow{2}{*}{$\begin{array}{c}\text { Con } \\
\text { Diarrea } \\
\mathbf{n}\end{array}$} & \multirow{2}{*}{$\begin{array}{c}\text { Sin } \\
\text { Diarrea } \\
\mathbf{n}\end{array}$} & \multirow{2}{*}{$\begin{array}{c}\text { Chi } 2 \\
\mathrm{P}<0,05\end{array}$} & \multicolumn{3}{|c|}{ Riesgo } \\
\hline & & & & OR & & \\
\hline & & & 0,105 & 5,536 & 0,609 & 50,311 \\
\hline Adecuado & 28 & 31 & & & & \\
\hline Inadecuado & 5 & 1 & & & & \\
\hline $\begin{array}{r}\text { Disposición de } \\
\text { excretas }\end{array}$ & & & $* 0,035$ & $\begin{array}{c}* 4,20 \\
3\end{array}$ & 1,035 & 17,069 \\
\hline Adecuado & 23 & 29 & & & & \\
\hline Inadecuado & 10 & 3 & & & & \\
\hline $\begin{array}{r}\text { Recolección de } \\
\text { basuras }\end{array}$ & & & 0,146 & 2,167 & 0,689 & 6,811 \\
\hline Adecuado & 22 & 26 & & & & \\
\hline Inadecuado & 11 & 6 & & & & \\
\hline $\begin{array}{r}\text { Sitio de preparación } \\
\text { de Alimentos }\end{array}$ & & & 0,746 & 1,032 & 0,062 & 17,24 \\
\hline Al aire libre & 1 & 1 & & & & \\
\hline $\begin{array}{r}\text { Cuarto Usado para } \\
\text { Cocinar }\end{array}$ & 32 & 31 & & & & \\
\hline $\begin{array}{r}\text { Material de la mesa } \\
\text { de manipulación de } \\
\text { alimentos }\end{array}$ & & & 0,182 & 1,96 & 0,642 & 5,894 \\
\hline Liso & 7 & 11 & & & & \\
\hline Poroso & 26 & 21 & & & & \\
\hline $\begin{array}{r}\text { Almacenamiento de } \\
\text { alimentos }\end{array}$ & & & 0,746 & 1,032 & 0,062 & 17,24 \\
\hline $\begin{array}{r}\text { Refrigerador y } \\
\text { recipientes }\end{array}$ & 32 & 31 & & & & \\
\hline Abierto al aire libre & 1 & 1 & & & & \\
\hline Aseo en la Vivienda & & & 0,201 & 0,494 & 0,145 & 1,679 \\
\hline Barre y Lava & 9 & 5 & & & & \\
\hline Barre y Trapea & 24 & 27 & & & & \\
\hline $\begin{array}{r}\text { Uso de detergentes y } \\
\text { desinfectantes en el } \\
\text { hogar }\end{array}$ & & & 0,488 & 2,133 & 0,184 & 24,761 \\
\hline SI & 32 & 30 & & & & \\
\hline No & 1 & 2 & & & & \\
\hline Material del piso & & & 0,52 & 1,25 & 0,304 & 5,147 \\
\hline Liso e impermeable & 28 & 28 & & & & \\
\hline Madera y Tierra & 5 & 4 & & & & \\
\hline $\begin{array}{r}\text { Material de las } \\
\text { paredes }\end{array}$ & & & 0,477 & 1,292 & 0,352 & 4,748 \\
\hline Bahareque y Madera & 6 & 5 & & & & \\
\hline Impermeable & 28 & 26 & & & & \\
\hline Material del Techo & & & 0,271 & 1,538 & 0,575 & 4,115 \\
\hline Concreto y Cemento & 20 & 16 & & & & \\
\hline Zinc y Desechos & 13 & 16 & & & & \\
\hline $\begin{array}{r}\text { Cocina separada de } \\
\text { otros espacios }\end{array}$ & & & *0,048 & 7,385 & 0,835 & 65,283 \\
\hline $\mathrm{Si}$ & 32 & 26 & & & & \\
\hline
\end{tabular}




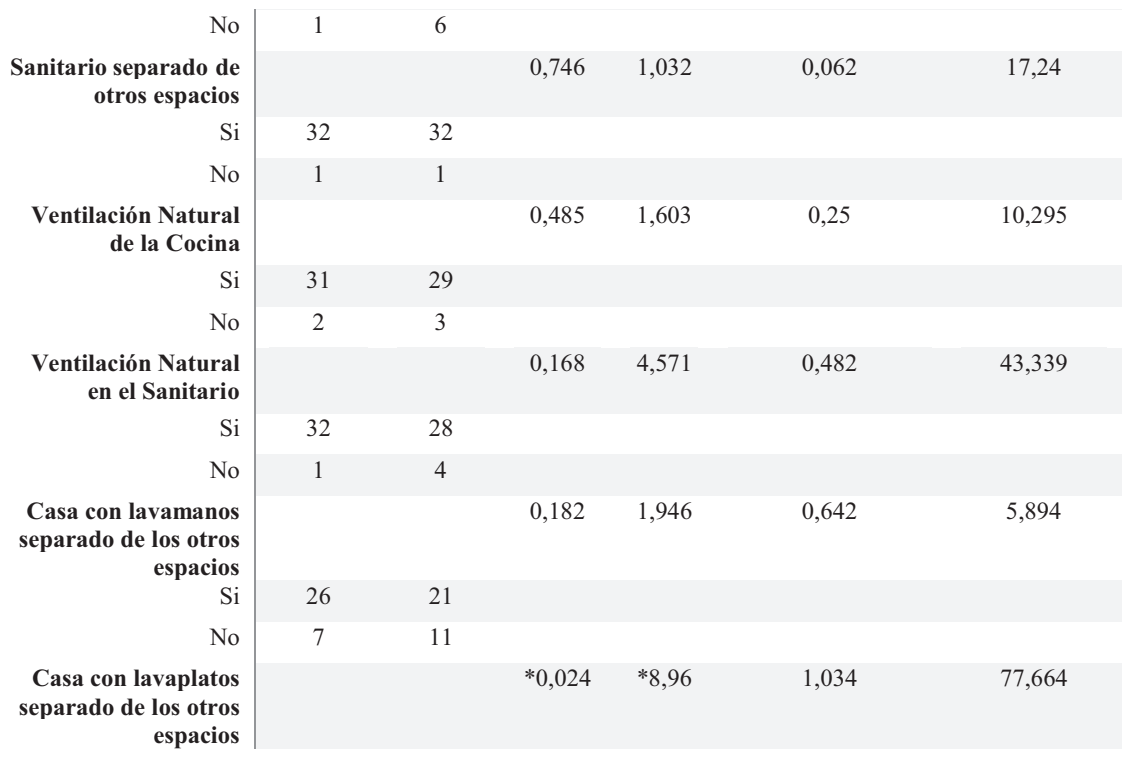

\section{Discusión}

De las 28 variables medidas tres de ellas correspondientes a si la vivienda tiene servicio de aseo, a la ubicación del tanque donde se almacena el agua y si la cocina se encuentra separada de otros espacios en el hogar tuvieron un $\mathrm{P}$ valor menor de 0.05 lo que permite afirmar que hay relación entre la aparición de diarrea y la falencia en el servicio de aseo, la mala ubicación del tanque y la no separación de la cocina con otros espacios del hogar. Valiente C, \& MoraD en el 2002 afirma que uno de los factores que incrementa los brotes de diarrea son los sedimentos de los tanques de almacenamiento y de la propia red de distribución del agua (14), además y en combinación del desabastecimiento de la fuente hídrica en concordancia con lo dicho por Díaz C en el 2013 (15), en el caso del Municipio de Caparrapí donde se observa un servicio intermitente del servicio de acueducto las personas optan por almacenar el agua en tanques, sin embargo en muchas ocasiones no lo instalan de manera adecuada con tapa y dentro de la vivienda o con techo, sino se dispone a las afueras de la casa, sin tapa o incluso se aprovecha en estos el almacenamiento de agua lluvia sin ningún tipo de tratamiento; en este estudio con un $\mathrm{P}$ valor de 0.014 la ubicación del tanque se considera un factor de riesgo para la aparición de diarreas en el hogar ( Tabla $3)$.

De otra parte la disposición de la cocina en el hogar en cuanto si está separada o no de las otras de las áreas del hogar se considera según Ros y colaboradores en el 2016 como uno de los factores actores Socio-Culturales y ambientales que impactan en las patologías prevenibles como anemia, diarrea, desnutrición infantil en niños de 0-4 años (16); esto se demuestra en el municipio de Caparrapí ya que esta variable en relación con la aparición de diarrea en la población de 0 a 15 años obtuvo un $\mathrm{P}$ valor de 0.048 lo que indica su relación directa con la aparición de episodios de diarrea en esta población, siguiendo esta misma línea cuando se evaluó el riesgo Odds ratio o tamaño del efecto a un intervalo de confianza al 95\%, el riesgo de que en las personas de 0 a 15 años de los hogares de Caparrapí presenten diarrea cuando no tienen separado el lavaplatos es significativamente mayor (8,96 veces) a los hogares que tienen separado el lavaplatos (Tabla 4).

En cuanto al manejo de excretas Hernández L y colaboradores en el 2011 afirmaron que las personas que habitan viviendas con sistemas de manejo de las aguas residuales deficientes presentaron exceso de riesgo de padecer diarreas, vómitos y otros síntomas asociados con la contaminación ambiental, y que además el mal manejo de los residuos 
tanto solidos como líquidos generan enfermedades en su población (18). Es importante anotar que la disposición de las excretas (P valor: 0,035; Odds radio: 4,203) tabla 4 y la presencia del servicio de aseo municipal en el hogar (P valor: 0,042) tabla 3 , estuvieron relacionadas con la aparición de diarrea o con el aumento del riesgo en contraerla, en el municipio se observaba que la disposición de excretas en algunas casas no se realiza de manera adecuada, en algunos lugares los inodoros se encentraban sin tapa y cerca de las fuentes de agua para consumo humano, lo que ocasiona que en esta población especificando la de 0-15 años de edad se favorezca la aparición de diarrea. Para el análisis de los datos se tomaron como variables las reportadas en la Tabla. 3 y 4 con esta información de 65 viviendas en donde habitaban personas de 0 a 15 años, se construyeron tablas de contingencia para determinar las frecuencias cruzando las variables de presencia o no de diarrea con las características sanitarias, hábitos de los hogares, para determinar cuál de estos factores tiene relación. Para determinar la relación se aplicó el estadístico Chi 2 usando el Software SPSS, se tomó como referencia el P valor, si este corresponde a un valor menor a 0.05 se acepta la hipótesis alternativa de que existe relación entre las variables y se rechaza hipótesis nula de que no existe relación entre las variables. De las 65 viviendas el $50.8 \%(\mathrm{n}=33)$ presentaron episodios de diarrea en los últimos 6 meses y el $49.2 \%(n=32)$ de las mismas no lo presentaron.

En cuanto al manejo de excretas Hernández L y colaboradores en el 2011 afirmaron que las personas que habitan viviendas con sistemas de manejo de las aguas residuales deficientes presentaron exceso de riesgo de padecer diarreas, vómitos y otros síntomas asociados con la contaminación ambiental, y que además el mal manejo de los residuos tanto solidos como líquidos generan enfermedades en su población (18). Es importante anotar que la disposición de las excretas (P valor: 0,035; Odds radio: 4,203) tabla 4 y la presencia del servicio de aseo municipal en el hogar ( $\mathrm{P}$ valor: 0,042$)$ tabla 3 , estuvieron relacionadas con la aparición de diarrea o con el aumento del riesgo en contraerla, en el municipio se observaba que la disposición de excretas en algunas casas no se realiza de manera adecuada, en algunos lugares los inodoros se encentraban sin tapa y cerca de las fuentes de agua para consumo humano, lo que ocasiona que en esta población especificando la de 0-15 años de edad se favorezca la aparición de diarrea.

Este estudio plantea la necesidad no solo de reconocer los factores predisponentes en la aparición de diarrea, ya que muchos de estos ya están presentes en la literatura; la necesidad radica en encontrar y comprobar estadísticamente cuales son los factores reales y puntuales que afectan a las comunidades de manera específica, para de esta manera intervenir directamente en el causal. De otro lado las limitaciones de este tipo de estudios radican en que, al ser municipios relativamente pequeños, no cuentan con el impacto y la visibilidad esperada a nivel nacional, sumado a que el acceso a la información por limitaciones geográficas y por decisión de la comunidad es limitado. De ahí la importancia de divulgar estudios de este tipo con el fin de encontrar posibles municipios con características similares en el mundo, que se puedan intervenir de manera equivalente.

\section{Conclusiones}

Se encontró que de las 349 personas encuestadas 116 corresponden a la edad de 0-15 años representando el 33\% de la población, de los 66 casos reportados de EDA en la población de 349,44 corresponden a la población de $0-15$ años es decir el $66.7 \%$ de la población infantil ha tenido diarrea en este periodo, lo que afirma que es la población más vulnerable. Las variables correspondientes a la presencia del servicio de aseo municipal en el hogar, ubicación del tanque de almacenamiento, disposición de la cocina separada de otros espacios en el hogar, lavaplatos separado de otros espacios y disposición de excretas fueron las que se relacionaron estadísticamente con la aparición de diarrea en la población de 0 a 15 años. 
En cuanto al manejo de excretas se observó que no en todas las casas se realiza de manera adecuada, en algunos lugares los inodoros se encuentran sin tapa y cerca de las fuentes de agua para consumo humano, al hablar de las basuras aunque tienen un servicio de aseo, en los hogares se observa basura distribuida a lo largo de la casa y en las calles del municipio no se observan suficientes canecas y al igual que en los hogares se ve basura arrojada en distintos sitios lo que aumenta el riesgo de adquirir plagas y microorganismos contaminantes.

El hecho de detectar específicamente cuál de todos los factores posibles son los que realmente están afectando a la población de Caparrapí permite crear medidas enfocadas a mitigar lo que en cierta medida aumenta el riesgo real de padecer diarrea en la población estudiada.

\section{Bibliografía}

1. Organización Mundial de la Salud.Cada año mueren 12,6 millones de personas a causa de la insalubridad del medio ambiente. Comunicado de prensa. 2016. Disponible en:http://www.who.int/mediacentre/news/releases/2016/deathsattributable-to-unhealthy-environments/es/

2. PrussUstun . A \&Corvanlán, C. (2006). Preventingdiseasethroughhealthyenviro nments. Organización Mundial de la Salud: Disponible en: http://www.who.int/ quantifying_ehimpacts/publications/preventingdisease.pdf

3. Instituto Nacional de Salud, Colombia. Informe de evento Mortalidad por EDA en menores de 5 años. 2012. Disponible en http://www.ins.gov.co/lineas-de-accion/ Subdireccion-Vigilancia/Informe\%20de\%20Evento\%20Epidemiolgico/EDA\%20 2012.pdf

4. Cáceres, D. C., Izquierdo, V. F., Mantilla, L., Jara, J., \&Velandia, M. Perfil epidemiológico de la población desplazada por el conflicto armado interno del país en un barrio de Cartagena, Colombia, 2000. Biomédica, 2002; 22:425-44.

5. Vázquez de Plata, G. E., Gómez de Avellaneda, E., \& Gamboa Delgado, E. M. Condiciones higiénico sanitarias de los servicios de alimentación en instituciones infantiles del Instituto Colombiano de Bienestar Familiar de Bucaramanga, Colombia. Revista Cubana de Alimentación y Nutrición. 2007; 17(1):23-33.

6. Alcaldía Municipal de Caparrapí. Plan de Desarrollo Municipal. Caparrapí.2012.

7. Municipio de Caparrapí. Plan Territorial de Salud 2008-2011. Caparrapí. 2008.

8. Organización Panamericana de la Salud/Organización Mundial de la Salud OPS/OMS. Representación en Colombia. (2010). Manual educativo Nacional. Hacia una vivienda saludable. Disponible en: http://www.paho.org/COL/index. php?option=com_content\&view $=$ article\&id=954:manual-educativo-nacionalhacia-una-vivienda-saludable-que-viva-mi hogar\&catid=681\&Itemid=361

9. Pereira, VV, Tibúrcio, DJ, Alves, SN \& da Silva, ES .Avaliação de parasitosesintestinais, estado nutricional e Indicadores sociaisemalunos de quatroescolas do ensino fundamental público da cidade de Divinópolis -Minas Gerais-Brasil. NeotropicalHelminthology, 2010; 4:149-157.

10. Organización Mundial de la Salud. Lucha contra las enfermedades transmitidas por el agua en los hogares. 2007. Disponible en :http://www.who.int/household_water/ advocacy/combating_disease_es.pdf

11. Organización Panamericana de la Salud. La salud en las Américas. Vo I. Publicación Científica y Técnica No. 587.2002. Disponible en: http://www.paho.org/hq/index. php?option $=$ com_docman\&task $=$ doc_view\&gid=16931\&Itemid $=$

12. Gardilcic F, Magdalena, Fica C, Alberto, Chang R, Mayling, Llanos M, Carolina, \&Luzoro V, Amaranta.Diarrea asociada a Clostridiumdifficile en un hospital de adultos.: Estudio descriptivo. Revista chilena de infectología. 2000;17(4): 307-312. 
13. Londoño, Ángela L. Mejía Shirley y Jorge E. Gómez-Marín. Prevalencia y Factores de Riesgo Asociados a Parasitismo Intestinal en Preescolares de Zona Urbana en Calarcá. Revista De Salud Pública. 2009;11 (1): 72-81.

14. Valiente C, \& Mora, D. El papel del agua para consumo humano en los brotes de diarrea reportados en el período 1999 - 2001 en Costa Rica. Revista Costarricense de Salud Pública. 2002; 11(20): 26-40.

15. Díaz Delgado Carlos. Agua potable para comunidades rurales, reusó y tratamientos avanzados de aguas residuales domésticas. Red Iberoamericana de Potabilización y Depuración del Agua. RIPDA-CYTED, Capítulo 13, Riesgo de enfermedades transmitidas por el agua en zonas rurales p 156. 2003.

16. Ros, $\mathrm{C}$ et al. Análisis de factores socio-culturales y ambientales involucrados en problemas de salud prevenibles (diarrea, malnutrición y anemia) en niños de 0 a 4 años en la comunidad de la Zona Sur de Gualeguaychú. Implementación y evaluación de intervenciones. Ciencia, Docencia y Tecnología Suplemento. 2016;6(6): 122-137.

17. Hernández, L, et al. Calidad del agua para consumo humano y salud: dos estudios de caso en Costa Rica. Revista Costarricense de Salud Pública. 2011; 20: 21-26. 